\title{
MUSIC FOR EXTENDED SCATTERERS AS AN INSTANCE OF THE FACTORIZATION METHOD*
}

\author{
TILO ARENS ${ }^{\dagger}$, ARMIN LECHLEITER $^{\ddagger}$, AND D. RUSSELL LUKE ${ }^{\S}$
}

\begin{abstract}
Recent work of Luke and Devaney showed that there exists an implementation of a modified linear sampling method that is equivalent to a MUSIC algorithm for scattering from soundsoft obstacles. The correspondence is independent of the size of the scatterer or the wavelength of the incident field. As the proof was not constructive, an explicit implementation could not be justified. In the present work, we show that MUSIC is an instance of the factorization method applied to any nonabsorbing scatterer, thus providing a justification for the MUSIC algorithm at arbitrary illuminating frequency for arbitrary nonabsorbing scatterers. These results are also extended to scattering from cracks. With explicit constructions in hand, we are also able to provide error and stability estimates for practical implementations in noisy environments with limited data and to explain a curious behavior of the factorization method in the case of noisy data.
\end{abstract}

Key words. inverse problems, scattering theory, factorization method, image processing, MUSIC

AMS subject classifications. 35R30, 35P25, 94A08

DOI. $10.1137 / 080737836$

1. Introduction. The multiple signal classification (MUSIC) algorithm is a well-known method for determining the location of emitters from sensors with arbitrary locations and arbitrary directional characteristics such as gain, phase, or polarization in a noise/interference environment [27]. The method is based on the observation that the far field patterns of point sources with centers near the true signal source are nearly orthogonal to the noise subspace of the far field operator, or the multistatic response matrix, as it is known in the signal and image processing literature. The connection between the MUSIC algorithm and other inverse scattering techniques has been explored by several authors [5, 16, 23]. Earlier studies are limited to point-like scatterers. More recent studies [11, 2, 12, 8, 9, 1] approach an application of the MUSIC algorithm to scatterers of some specified size, relative to the wavelength, and are based on the finite-dimensional multistatic response matrix for point-like scatterers. An anonymous referee brought to our attention [3], which deals with closely related questions for linearized scattering. In [23], Luke and Devaney combine the linear sampling method of Colton and Kirsch [6] with the point source method of Potthast [26] to show the existence of a field that is orthogonal to the far field patterns of point sources centered on the boundary of the scatterers. This extends the MUSIC algorithm to obstacles of arbitrary size, independent of the frequency. Missing from their analysis, however, is a concrete way to construct such a field. Numerical experiments indicate that fields constructed from elements of the noise subspace of the far field operator (that is, the subspace corresponding

\footnotetext{
*Received by the editors October 12, 2008; accepted for publication (in revised form) July 22, 2009; published electronically October 22, 2009.

http://www.siam.org/journals/siap/70-4/73783.html

${ }^{\dagger}$ Fakultät für Mathematik, Universität Karlsruhe, 76128 Karlsruhe, Germany (arens@numathics. com).

${ }^{\ddagger}$ INRIA Futurs Saclay / CMAP, Ecole Polytechnique, 91128 Palaiseau, France (alechle@cmapx. polytechnique.fr).

$\S$ Department of Mathematical Sciences, University of Delaware, Newark, DE 19716-2553 (rluke@ math.udel.edu). This author's work was supported in part by NSF grant DMS-0712796.
} 
to small eigenvalues of the far field operator) yield the desired properties. In the present work we use Kirsch's factorization method [14] to prove this to be the case, thus yielding a constructive proof of the MUSIC algorithm for extended obstacles. Moreover, our analysis is not limited to Dirichlet obstacles, but rather to scatterers whose corresponding far field operators are normal. In particular, this is the case for nonabsorbing scatterers.

Our formalism begins with exact measurements of the far field of a scatterer embedded in a homogeneous medium and illuminated by a single frequency plane wave. Which frequency one uses for the incident field is of no consequence to the analysis or the method, though in practice this may be an important consideration due to instrumentation limitations. Also, readers accustomed to incident pulses rather than fields will note the conspicuous absence of multiple frequencies. We do not address the issues of multiple frequency data, since the method can be applied independently to each incident field frequency so long as these are distinct. Readers are referred to $[22,24]$ for a discussion of how one combines single frequency reconstructions from multiple frequency data. Limiting our focus to only single frequency fields also allows us to treat two- and three-dimensional scattering at the same time, since our formalism applies equally to each setting. As discussed in [22], this is not the case for multiple frequency data. Our simulations are in two dimensions but can be applied with equal ease in three dimensions, the main difficulty being in the simulation of the far field data rather than with the reconstruction.

We do not explicitly take into account clutter or instrument noise. Instead we interpret noise generically in terms of the size of the spectrum of the far field measurements relative to that of the systematic error, whatever its source. The point of the analysis, however, is that we need not be concerned with the nature of the noise, just its size. The key to our result is to relate the indicator function, which is used in the factorization method to characterize the scatterer, to the value of a Herglotz wave function with a specially chosen density. We show that this Herglotz wave function is, roughly speaking, small on the obstacle and large in some region outside. Taking a different perspective, the special densities we use are arbitrarily close to being orthogonal to the data space of the far field operator, consisting of the first few eigenvalues that can be measured with reliable precision. Typically, the data subspace consists of eigenfunctions with associated eigenvalues above the noise level. The latter observation concerning orthogonality is also clear from the construction of our special densities within the noise space of the operator. This subspace is spanned by those eigenfunctions that cannot be measured reliably-typically, the space spanned by those eigenfunctions of the order of or below the noise level. Any measurement of the far field operator will not faithfully reproduce these eigenfunctions but rather strongly perturbed versions of them. However, these will always be orthogonal to the data subspace. This crucial property is the basis for our analysis in the case of perturbed data arising either from a discretization or measurement error.

Our approach also gives rise to the construction of special entire solutions to the Helmholtz equation which avoid the scatterer and hence produce little scattering. For this reason, they are called nonscattering fields in [23]. We provide some numerical examples of such functions.

The main results of the paper are contained in sections 3 and 5 . The preceding section 2 provides the necessary mathematical and notational background. In section 3 we state our result on the MUSIC algorithm for extended scatterers, and construct nonscattering fields. Section 4 contains equivalent results in the case of scattering by a crack. In this case, the analysis of the factorization method requires more 
sophisticated arguments, as such scatterers cannot be characterized using the far field patterns of point sources. Section 5 is devoted to analyzing the stability of these constructions in the case of a perturbed far field operator. All these results are valid for scattering problems in which the far field operator is normal. We use scattering by a sound-soft obstacle and by a penetrable obstacle as numerical examples. We conclude with section 6 , where we use some of the derived estimates to explain a curious behavior of the factorization method in the case of noisy data. The appendix of the paper is devoted to establishing the relation of the spectral properties of the continuous far field operator to those of the discrete version used implicitly throughout the literature in numerical examples.

2. Direct and inverse scattering background. We consider acoustic scattering of small-amplitude, monochromatic, and time-harmonic waves from one or more scatterers embedded in an isotropic homogeneous medium. The scatterers are identified by the domain $D \subset \mathbb{R}^{m}, m=2$ or 3 , which is assumed to be a bounded Lipschitz domain with connected exterior and boundary $\partial D$; the unit outward normal to $\partial D$ is denoted by $\nu$. Our model to mathematically describe wave propagation outside the scatterer $D$ is the Helmholtz equation,

$$
\left(\Delta+k^{2}\right) u(x)=0, \quad x \in \mathbb{R}^{m} \backslash \bar{D},
$$

where $\Delta$ denotes the Laplacian and $k>0$ is the wavenumber. We denote by $u^{i}(x, \hat{\eta})=$ $\exp (\mathrm{i} k \hat{\eta} \cdot x)$ an incident plane wave of direction $\hat{\eta} \in \mathbb{S}:=\left\{\hat{x} \in \mathbb{R}^{m}:|\hat{x}|=1\right\}$ that causes a scattered field $u^{s}(\cdot, \hat{\eta})$ satisfying $(2.1)$ and the Sommerfeld radiation condition

$$
r^{\frac{m-1}{2}}\left(\frac{\partial}{\partial r}-i k\right) u^{s}(x, \hat{\eta}) \rightarrow 0 \quad \text { as } r=|x| \rightarrow \infty,
$$

uniformly in all directions $\hat{x}=x /|x|$. We note that Sommerfeld's radiation condition implies that the scattered field has the behavior

$$
u^{s}(x, \hat{\eta})=\frac{e^{\mathrm{i} k|x|}}{|x|^{\frac{m-1}{2}}}\left\{u^{\infty}(\hat{x}, \hat{\eta})+O\left(|x|^{-1}\right)\right\} \quad \text { as }|x| \rightarrow \infty
$$

where the function $u^{\infty}$ is called the far field pattern of $u^{s}$; see, e.g., [7].

Of course, incident and scattered fields are coupled via conditions describing the physical nature of the scatterer. We discuss in this section two different kinds of scatterers: impenetrable sound-soft obstacles and penetrable media. For a sound-soft obstacle, the total field $u: \mathbb{R}^{m} \backslash D \rightarrow \mathbb{C}$ given by $u=u^{i}+u^{s}$ satisfies a homogeneous Dirichlet boundary condition $u(x)=0$ for $x \in \partial D$.

It is well known that this scattering problem is uniquely solvable (see, e.g., [25]). Consequently, there is a unique scattered field $u^{s} \in H_{\mathrm{loc}}^{1}\left(\mathbb{R}^{m} \backslash \bar{D}\right)$ which is a weak solution of (2.1) outside of $\bar{D}$ and satisfies the boundary condition $u^{s}=-u^{i}$ in the trace sense of $H^{1 / 2}(\partial D)$. More generally, there exists a bounded data-to-pattern operator $G_{\mathrm{D}}: H^{1 / 2}(\partial D) \rightarrow L^{2}(\mathbb{S})$ which maps the Dirichlet boundary data on $\partial D$ to the far field pattern of the corresponding radiating solution of the Helmholtz equation.

Let us recall the outgoing free-space fundamental solution to (2.1),

$$
\Phi(x, y):=\frac{\mathrm{i}}{4} H_{0}^{(1)}(k|x-y|), \quad m=2, \quad \Phi(x, y):=\frac{1}{4 \pi} \frac{e^{\mathrm{i} k|x-y|}}{|x-y|}, \quad m=3,
$$


both for $x \neq y$. Here $H_{0}^{(1)}$ denotes the zeroth-order Hankel function of the first kind. Each $\Phi(\cdot, y)$ satisfying the Sommerfeld radiation condition is associated with a far field pattern, denoted $\Phi^{\infty}(\cdot, z)$ and given by [7],

$$
\Phi^{\infty}(\hat{x}, z)=\beta e^{-\mathrm{i} k \hat{x} \cdot z}, \quad \hat{x} \in \mathbb{S},
$$

where $\beta=e^{\mathrm{i} \frac{\pi}{4}} / \sqrt{8 \pi k}$ for $m=2$ and $\beta=1 /(4 \pi)$ for $m=3$.

A linear superposition of plane waves by a density $g$ yields the Herglotz wave function $v_{g}$,

$$
v_{g}(x):=\int_{\mathbb{S}} e^{\mathrm{i} k \widehat{\eta} \cdot x} g(\widehat{\eta}) d s(\widehat{\eta}) \quad \text { for } x \in \mathbb{R}^{m} .
$$

Obviously, $v_{g}$ satisfies the Helmholtz equation (2.1) in all of $\mathbb{R}^{m}$. Since the Helmholtz equation is a linear partial differential equation, the superposition principle states that the far field pattern to a linear combination of incident plane waves $u^{i}(x, \widehat{\eta})$ is the corresponding linear combination of scattered fields $u^{s}(x, \widehat{\eta})$. The far field operator

$$
F: L^{2}(\mathbb{S}) \rightarrow L^{2}(\mathbb{S}), \quad F g:=\int_{\mathbb{S}} u^{\infty}(\cdot, \widehat{\eta}) g(\widehat{\eta}) d s(\widehat{\eta}),
$$

captures this observation, mapping a density $g \in L^{2}(\mathbb{S})$ onto the far field of the scattered field generated by the incident Herglotz wave function $v_{g}$. Sometimes we will need to distinguish far field operators for different types of scattering problems. We will then write $F_{\mathrm{D}}$ instead of $F$, with the subscript D referring to the homogeneous Dirichlet boundary condition satisfied by the total field.

In addition to obstacles, we will also consider penetrable scatterers consisting of an inhomogeneous medium, where the inhomogeneity is modeled by a nonnegative real refractive index $n^{2}$ with $n^{2}=1$ on $\mathbb{R}^{m} \backslash \bar{D}$. The contrast $q$ is the difference between the background medium and the refractive index, $q=n^{2}-1$ in $\mathbb{R}^{m}$. Obviously, $q$ is compactly supported. We assume that $D$ is the support of $q$ - even more, that

$$
q \in L^{\infty}(D) \quad \text { and } \quad q \geq c_{0}>0 \quad \text { in } D .
$$

In particular, $q$ is assumed to be real-valued. In a slight abuse of notation, we do not distinguish between $q$ and its extension to all of $\mathbb{R}^{m}$ by zero outside $D$.

Consider now scattering of a plane wave $u^{i}(\cdot, \widehat{\eta})$ by $D$. The total field $u=u^{i}+u^{s}$ satisfies $\left(\Delta+k^{2}(1+q)\right) u(\cdot, \widehat{\eta})=0$ in $\mathbb{R}^{m}$, and hence the scattered field $u^{s}(\cdot, \widehat{\eta})$ satisfies the inhomogeneous Helmholtz equation

$$
\left(\Delta+k^{2}(1+q)\right) u^{s}(\cdot, \widehat{\eta})=-k^{2} q u^{i}(\cdot, \widehat{\eta}), \quad x \in \mathbb{R}^{m} \backslash \partial D, \widehat{\eta} \in \mathbb{S},
$$

in addition to the Sommerfeld radiation condition (2.2). Under our assumptions, this source problem has a unique solution in $H_{\mathrm{loc}}^{1}\left(\mathbb{R}^{m}\right)$ for any $f=-k^{2} q u^{i}(\cdot, \widehat{\eta}) \in L^{2}(D)$ [7]. Moreover, the data-to-pattern operator $G_{\mathrm{M}}: L^{2}(D) \rightarrow L^{2}(\mathbb{S})$, which maps $f$ to the far field pattern of the radiating solution to this source problem, is bounded.

Replacing again the individual plane wave $u^{i}(\cdot, \widehat{\eta})$ with the superposition $v_{g}$, the far field operator is formally defined by (2.4). If we need to stress the dependence on the type of scattering problem, we will write $F_{\mathrm{M}}$ instead of $F$. Since the contrast $q$ is real-valued, the medium is nonabsorbing and the far field operator is normal, an important prerequisite for the later analysis. 
We now shift our attention to the inverse scattering problems of reconstruction of the scatterer's support from knowledge of $F$. In [14] the factorization method was introduced as a simple and explicit method to reconstruct the scatterer's support from spectral data of $F$. Although various sophisticated extensions of this method exist which make it applicable to cases where $F$ is nonnormal (see [17] and the references therein), we consider here only the original version of the method presented in [14] for the case of a normal far field operator. Another necessary ingredient is that $F$ is injective with dense range, which is assured by the following assumptions.

Assumption 2.1.

(a) When dealing with the Dirichlet scattering problem, we assume that $k^{2}$ is not a Dirichlet eigenvalue of $-\Delta$ in $D$.

(b) Concerning the medium scattering problem, we assume that $k^{2}$ is not an interior transmission eigenvalue of $D$. This means that there are no nontrivial weak solutions $(u, v) \in H^{2}(D) \times H^{2}(D)$ to

$$
\Delta u+k^{2}(1+q) u=0 \quad \text { and } \quad \Delta v+k^{2} v=0 \quad \text { in } D
$$

with $u=v$ on $\partial D$ and $\frac{\partial u}{\partial \nu}=\frac{\partial w}{\partial \nu}$ on $\partial D$.

The idea of the factorization method is to characterize $D$ via the range of the square root of $F$. Kirsch proved in $[14,15]$ that the range $\mathcal{R}\left(\left(F^{*} F\right)^{1 / 4}\right)$ equals the range of the data-to-pattern operator $G$ and exploited the fact that the far field pattern $\Phi^{\infty}(\cdot, z)$ of a point source at $z$ is in the range of $G$ precisely if $z$ is inside the obstacle. We collect the statement of the factorization method for the two scattering problems under investigation in the following theorem, which is shown in $[17,14,15]$.

TheOrem 2.2 (factorization method). We assume that Assumption 2.1 holds.

1. The data-to-pattern operator $G_{\mathrm{D}}: H^{1 / 2}(\partial D) \rightarrow L^{2}(\mathbb{S})$ is a compact, injective, bounded linear operator with dense range. $\mathcal{R}\left(G_{\mathrm{D}}\right)=\mathcal{R}\left(\left(F_{\mathrm{D}}^{*} F_{\mathrm{D}}\right)^{1 / 4}\right)$, and $\left(F_{\mathrm{D}}^{*} F_{\mathrm{D}}\right)^{-1 / 4} G_{\mathrm{D}}$ is a norm isomorphism from $H^{1 / 2}(\partial D)$ onto $L^{2}(\mathbb{S})$.

2. The data-to-pattern operator $G_{M}: L^{2}(D) \rightarrow L^{2}(\mathbb{S})$ is compact with dense range. Let $\mathcal{H}$ be the closure of $\left\{v \in C^{\infty}(\bar{D}), \Delta v+k^{2} v=0\right\}$ in $L^{2}(D)$, and denote by $\left.G\right|_{\mathcal{H}}$ the restriction of $G$ to $\mathcal{H}$. Then $\mathcal{R}\left(\left.G\right|_{\mathcal{H}}\right)=\mathcal{R}\left(\left(F^{*} F\right)^{1 / 4}\right)$, and $\left(F_{\mathrm{M}}^{*} F_{\mathrm{M}}\right)^{-1 / 4} G_{\mathrm{M}}$ is a norm isomorphism from $\mathcal{H}$ onto $L^{2}(\mathbb{S})$.

In either case, the far field pattern $\Phi^{\infty}(\cdot, z)$ of the fundamental solution to the Helmholtz equation is in the range of the data-to-pattern operator if and only if $z \in D$.

In the following sections we will at various stages present two-dimensional numerical examples. Since we are not using multiple frequencies, there is no qualitative difference between scattering in two and three dimensions; the only significant numerical difference is in the simulation of the far field data, not in the inversion algorithm. Here, the direct scattering problem is solved for $J$ directions of incidence distributed uniformly on the unit circle, $\hat{x}_{j}=(\cos (2 \pi j / J), \sin (2 \pi j / J))^{\top}, j=0, \ldots, J-1$, and the far field pattern of the scattered field is determined for those same $J$ directions. Using this data, the far field operator is approximated by replacing the integral by an order $J$ composite rectangular rule. In other words, a discrete linear map $F_{\mathrm{FD}}: \mathbb{C}^{J} \rightarrow \mathbb{C}^{J}$ (FD short for fully discrete) is available where, for $\xi \in \mathbb{C}^{J}$,

$$
\left(F_{\mathrm{FD}} \xi\right)_{j}=\frac{2 \pi}{J} \sum_{k=0}^{J-1} u^{\infty}\left(\hat{x}_{j}, \hat{x}_{k}\right) \xi_{k}, \quad j=0, \ldots, J-1 .
$$

Though it is rarely discussed in the literature, it is not obvious in what sense the spectral data of the matrix $F_{\mathrm{FD}}$ approximates the spectral data of the continuous 
operator $F$. In the appendix, we investigate the relation of $F_{\mathrm{FD}}$ to $F$. We show that there is a semidiscrete operator $F_{\mathrm{SD}}: L^{2}(\mathbb{S}) \rightarrow L^{2}(\mathbb{S})$ with the same nontrivial eigenvalues as $F_{\mathrm{FD}}$ which is close in norm to $F$. Arguments from operator perturbation theory then show that the first few eigenvalues and eigenspaces of $F_{\mathrm{FD}}$ are good approximations to those of $F$.

3. Nonscattering fields and the MUSIC algorithm for extended scatterers. We will now show that from knowledge of the far field operator it is possible to construct entire solutions of the Helmholtz equation in $\mathbb{R}^{m}$ that are arbitrarily small inside the scatterer. Moreover, there is always a region outside the scatterer where these fields are large; i.e., these fields are nontrivial. By construction, these fields are Herglotz wave functions with densities orthogonal to those first few eigenspaces corresponding to the largest eigenvalues of $F$ which can be measured with reliable precision. As we discussed in the introduction, we call the span of these spaces the signal space and its orthogonal complement the noise space, in analogy to the terminology used in connection with the MUSIC algorithm.

By the spectral theorem for compact normal operators the far field operator $F$ admits a system of eigenfunctions that form a complete orthonormal basis of $L^{2}(\mathbb{S})$ and whose corresponding eigenvalues have a unique cluster point at 0 . Let $\left(\lambda_{n}, \xi_{n}\right)$ for $n \in \mathbb{N}$ be the system of eigenvalues and orthonormal eigenvectors of $F$ with eigenvalues $\left|\lambda_{n}\right| \geq\left|\lambda_{m}\right|$ for $m>n$. For $x, z \in \mathbb{R}^{m}$ let $M, N \in \mathbb{N}$ with $M \leq N$, and define

$$
g_{M, N, z}:=\sum_{n=M}^{N} \frac{1}{\left|\lambda_{n}\right|^{1 / 2}}\left\langle\Phi^{\infty}(\cdot, z), \xi_{n}\right\rangle \xi_{n} \quad \text { in } L^{2}(\mathbb{S}) .
$$

Note that $\left(F^{*} F\right)^{1 / 4} g_{M, N, z}=P_{M N} \Phi^{\infty}(\cdot, z)$, where $P_{M N}$ is the orthogonal projection of $L^{2}(\mathbb{S})$ onto $\operatorname{span}\left\{\xi_{M}, \xi_{M+1}, \ldots, \xi_{N}\right\}$. Define the density

$$
\hat{g}_{M, N, z}:=\frac{1}{\left\|g_{M, N, z}\right\|_{L^{2}(\mathbb{S})}} \sum_{n=M}^{N} \frac{1}{\left|\lambda_{n}\right|}\left\langle\Phi^{\infty}(\cdot, z), \xi_{n}\right\rangle \xi_{n} .
$$

In [23] it was proved that there exists a nontrivial density in the noise space of the far field operator. We show next that the density $\hat{g}_{M, N, z}$ yields an explicit approximation of such a density. Indeed, (2.3) and (3.2) yield the following identity:

$$
\begin{aligned}
v_{\hat{g}_{M, N, z}}(x) & =\int_{\mathbb{S}} e^{\mathrm{i} k \hat{\eta} \cdot x} \hat{g}_{M, N, z}(\widehat{\eta}) d s(\widehat{\eta}) \\
& =\frac{1}{\beta\left\|g_{M, N, z}\right\|_{L^{2}(\mathbb{S})}} \sum_{n=M}^{N} \frac{1}{\left|\lambda_{n}\right|}\left\langle\Phi^{\infty}(\cdot, z), \xi_{n}\right\rangle \overline{\left\langle\Phi^{\infty}(\cdot, x), \xi_{n}\right\rangle} .
\end{aligned}
$$

TheOrem 3.1. Under Assumption 2.1 we have that for given $\epsilon>0$ and given compact domains $K_{-} \subset D$ and $K_{+} \subset \mathbb{R}^{m} \backslash D$ there are $M, N \in \mathbb{N}$ with $0<M<N$ such that

$$
\left|v_{\hat{g}_{M, N, z}}(z)\right|<\varepsilon \quad \text { for } z \in K_{-} \quad \text { and } \quad\left|v_{\hat{g}_{M, N, z}}(z)\right|>\frac{1}{\varepsilon} \quad \text { for } z \in K_{+},
$$

where $v_{\hat{g}_{M, N, z}}(x)$ is defined by (3.3). 
Proof. For any $z \in \mathbb{R}^{m}, M, N \in \mathbb{N}$ such that $0<M<N$, we have

$$
\begin{aligned}
\left|v_{\hat{g}_{M, N, z}}(z)\right| & =\frac{1}{\left\|g_{M, N, z}\right\|_{L^{2}(\mathbb{S})}}\left|\frac{1}{\beta} \sum_{n=M}^{N} \frac{1}{\left|\lambda_{n}\right|}\left\langle\Phi^{\infty}(\cdot, z), \xi_{n}\right\rangle \overline{\left\langle\Phi^{\infty}(\cdot, z), \xi_{n}\right\rangle}\right| \\
& =\frac{1}{\left\|g_{M, N, z}\right\|_{L^{2}(\mathbb{S})}} \frac{1}{|\beta|} \sum_{n=M}^{N} \frac{1}{\left|\lambda_{n}\right|}\left|\left\langle\Phi^{\infty}(\cdot, z), \xi_{n}\right\rangle\right|^{2}=\frac{1}{|\beta|}\left\|g_{M, N, z}\right\|_{L^{2}(\mathbb{S})} .
\end{aligned}
$$

Consider first $z \in K_{-} \subset D$. From Theorem 2.2, we know that

$$
v_{\hat{g}_{M, \infty, z}}(z):=\lim _{N \rightarrow \infty} v_{\hat{g}_{M, N, z}}(z)
$$

is bounded on $K_{-}$, and $v_{\hat{g}_{M, \infty}, z}(z) \rightarrow 0$ uniformly on $K_{-}$as $M \rightarrow \infty$. Uniformity is due to Dini's theorem, because the convergence $\left\|g_{M, \infty, z}\right\|_{L^{2}(\mathbb{S})} \rightarrow 0$ as $M \rightarrow \infty$ is monotonic for each $z \in D$. Hence by choosing $M$ large enough, we obtain the upper bound on $K_{-}$.

Similarly, for $z \in K_{+}$, we know that $\left\|g_{M, N, z}\right\|_{L^{2}(\mathbb{S})}$ is unbounded as $N \rightarrow \infty$. Again using Dini's theorem for $1 /\left\|g_{M, \infty, z}\right\|_{L^{2}(\mathbb{S})}$, we obtain uniformity on the compact domain $K_{+}$and hence the lower estimate by choosing $N$ appropriately.

We remark that

$$
W_{M N}(z):=\left|\beta v_{\hat{g}_{M, N, z}}(z)\right|^{2}=\sum_{n=M}^{N} \frac{1}{\left|\lambda_{n}\right|}\left|\left\langle\Phi^{\infty}(\cdot, z), \xi_{n}\right\rangle\right|^{2}
$$

is simply a partial sum of the Picard series in the factorization method; moreover, up to weighting, it is the indicator computed in the MUSIC algorithm. A simple algorithm for imaging scatterers is immediately suggested: for a predetermined $M$ and $N$ evaluate $W_{M N}(z)$ at points $z_{j}$ on a grid in some computational domain. The scatterer is estimated as those points where $W_{M N}\left(z_{j}\right)$ is small.

As with many inverse scattering algorithms (e.g., the linear sampling method, the factorization method, and the point source method) one has to choose a cutoff value for the sampling function (3.6). Also, one must be able to choose $M$ and $N$ large enough to observe the desired behavior. Choosing a cutoff and the parameters $M$ and $N$ would be part of a calibration process that must be performed in any practical implementation. In particular, if there is substantial noise in the far field measurement, it is unrealistic to expect that such an $M$ and $N$ can be chosen to achieve reasonable contrast. However, we show in the next section that any density orthogonal to the signal space of $F$ will yield a small amplitude Herglotz wave function on closed subsets of the scatterer. In contrast, for $z \in \mathbb{R}^{m} \backslash \bar{D}$ there is no guarantee that an arbitrary density in the space orthogonal to the signal space will generate the desired blow-up. In other words, a blow-up of the indicator function (3.4) or (3.6) will generate a true positive, though the absence of such a blow-up is uninformative. The key point for stability is that we generate $\widehat{g}_{M, N, z}$ in the noise subspace of $F$, and the only property we really exploit is that $\widehat{g}_{M, N, z}$ is in the orthogonal complement of the data subspace. This property remains true under perturbation, even if the eigenspaces themselves are strongly perturbed. (Note that in general the perturbed operator will not possess eigenvalues.)

A slight variation of the proof of Theorem 3.1 yields fields that are small on the scatterer while large at least in some compact set outside. For the formulation of this theorem we introduce the notation $B_{\delta}(z)$ for an open ball of radius $\delta$ centered at $z$. 
TheOREm 3.2 (nonscattering fields). Let Assumption 2.1 be satisfied. Given any $\epsilon>0, K_{-} \subset D$ closed, and $K_{+} \subset \mathbb{R}^{m} \backslash D$ compact, there exist $M, N \in \mathbb{N}$ and $\delta>0$ such that

$$
\left|v_{\hat{g}_{M, N, z}}(x)\right|<\epsilon, \quad x \in K_{-}, z \in \mathbb{R}^{m}, \quad \text { and } \quad\left|v_{\hat{g}_{M, N, z}}(x)\right|>\frac{1}{\epsilon}, \quad z \in K_{+}, x \in B_{\delta}(z) .
$$

Proof. Generalizing the approach in the proof of Theorem 3.1, we estimate

$$
\begin{aligned}
\left|v_{\hat{g}_{M, N, z}}(x)\right| \leq & \frac{1}{|\beta|\left\|g_{M, N, z}\right\|_{L^{2}(\mathbb{S})}} \sum_{n=M}^{N} \frac{1}{\left|\lambda_{n}\right|}\left|\left\langle\Phi^{\infty}(\cdot, z), \xi_{n}\right\rangle \overline{\left\langle\Phi^{\infty}(\cdot, x), \xi_{n}\right\rangle}\right| \\
\leq & \frac{1}{|\beta|\left\|g_{M, N, z}\right\|_{L^{2}(\mathbb{S})}}\left(\sum_{n=M}^{N} \frac{1}{\left|\lambda_{n}\right|}\left|\left\langle\Phi^{\infty}(\cdot, z), \xi_{n}\right\rangle\right|^{2}\right)^{1 / 2} \\
& \times\left(\sum_{n=M}^{N} \frac{1}{\left|\lambda_{n}\right|}\left|\left\langle\Phi^{\infty}(\cdot, x), \xi_{n}\right\rangle\right|^{2}\right)^{1 / 2} \\
\leq & \frac{1}{|\beta|}\left(\sum_{n=M}^{N} \frac{1}{\left|\lambda_{n}\right|}\left|\left\langle\Phi^{\infty}(\cdot, x), \xi_{n}\right\rangle\right|^{2}\right)^{1 / 2}=\frac{1}{|\beta|}\left\|g_{M, N, x}\right\|_{L^{2}(\mathbb{S})} .
\end{aligned}
$$

As this upper bound is independent of $z$, we obtain the upper bound for $x \in K_{-}$and $z \in \mathbb{R}^{m}$ exactly as in the proof of the previous theorem.

On the other hand, if $x=z \in K_{+}$, we directly use (3.5). Since $z \notin D$, by Theorem 2.2 we have that $\left\|g_{M, N, z}\right\|_{L^{2}(\mathbb{S})}$ becomes arbitrarily large as $N \rightarrow \infty$ uniformly in $z \in K_{+}$. We choose $N$ such that $\left|v_{\hat{g}_{M, N, z}}(z)\right|>2 / \epsilon$ for $z \in K_{+}$. As $v_{\hat{g}_{M, N, z}}(x)$ is continuous with respect to both $x$ and $z$ and $K_{+}$is compact, there is a radius $\delta$ such that for any $z \in K_{+}$and $x \in B_{\delta}(z)$ we have $\left|v_{\hat{g}_{M, N, z}}(x)\right|>1 / \epsilon$.

REMARK 3.3. The results of Theorems 3.1 and 3.2 are independent of the type of the scattering problem and hold whenever the far field operator is normal, for instance for obstacles with a Neumann boundary condition. The crucial ingredient is the behavior of the Picard series in the factorization method, and this is independent of the type of scattering problem as long as the corresponding far field operator is normal.

For imaging purposes, Theorem 3.2 suggests plotting the Herglotz wave function $v_{\hat{g}_{M, N, z}}$. The scatterer will be contained in the set where this function is small. If $z$ is located outside the scatterer, the function will also be large for $x$ close to $z$.

In [23], numerical examples are shown for a MUSIC algorithm for extended scatterers, with the Herglotz wave functions used in the indicator constructed by trial and error. Here, we report similar results using our constructive approach. The reconstructions obtained are similar to those reported for the factorization method.

As a numerical example, we consider scattering by two sound-soft kite-shaped obstacles positioned in two-dimensional free space; Figure 1(a) shows the configuration. The wavenumber in this example is $k=1$.

The scattering problem was solved numerically using a boundary integral equation method for 25 incident plane waves with directions $\hat{x}_{j}, j=0, \ldots, 24$, uniformly distributed on $[-\pi, \pi]$; the far field pattern was sampled in those same directions to obtain a corresponding operator $F_{\mathrm{FD}}$ (see the end of section 2). In Figure 1(b) the singular values of $F_{\mathrm{FD}}$ are displayed. We have high confidence that these singular values are extremely good approximations of the absolute values of the 25 largest eigenvalues of $F$. As a test, we computed $F_{\mathrm{FD}}$ with twice the number of incident and 


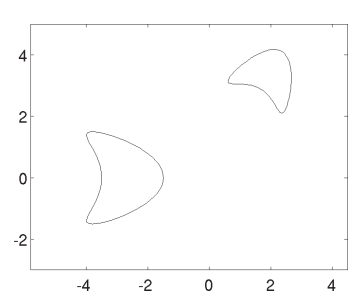

(a)

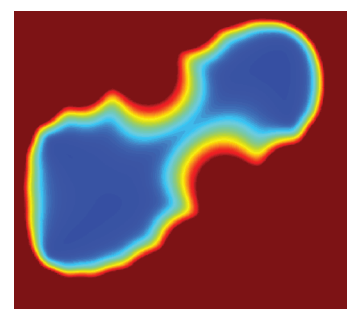

(d)

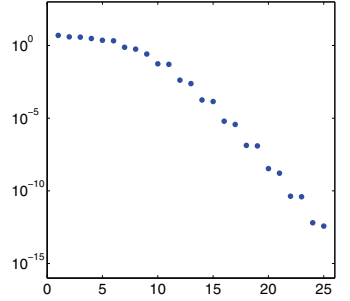

(b)

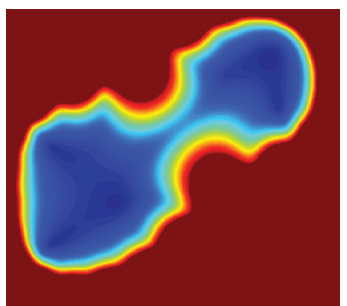

(e)

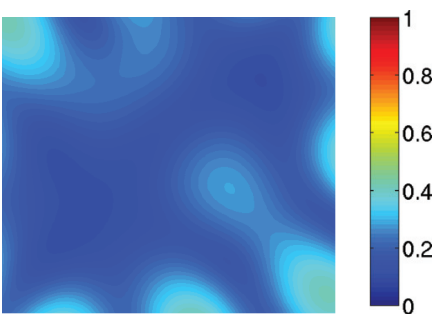

(c)

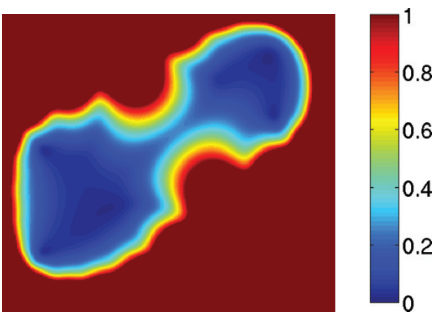

(f)

FIG. 1. (a) Configuration for the example problem. (b) Singular values of $F_{\mathrm{FD}}$ generated from 25 incident plane waves with directions uniformly distributed on $[-\pi, \pi]$. (c)-(f) Plots of the MUSIC indicator $W_{M N}$ for (c) $M=1, N=10$; (d) $M=1, N=25$; (e) $M=8, N=25$; (f) $M=12$, $N=25$.

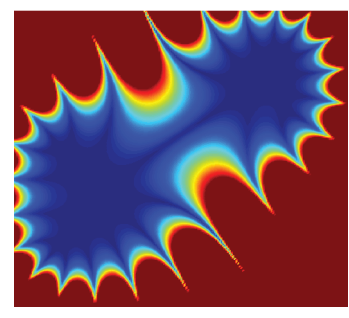

(a) $z=(0,0)^{\top}$

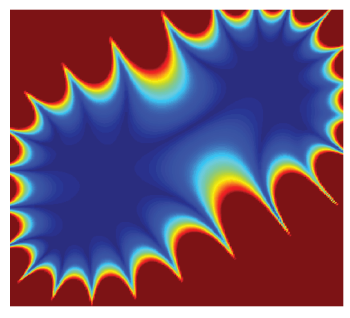

(b) $z=(-2.5,0)^{\top}$

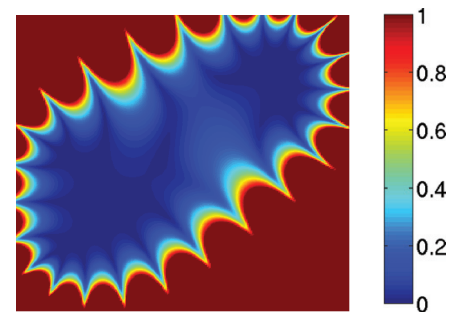

(c) $z=(20,0)^{\top}$

FIG. 2. Absolute values of nonscattering fields for $M=22, N=25$, and various locations of $z$.

observation directions and obtained the same values for the first 25 singular values up to 16-digit numerical precision.

In Figure 1(c)-(f) we show the value of the indicator $W_{M N}$ for various values of $M$ and $N$ plotted over points on a grid. Values of $W_{M N}(z)>1$ are plotted with the same color. As can be seen, increasing $N$ is indeed responsible for larger values of the indicator outside the obstacle. Increasing $M$ visibly reduces $W_{M N}(z)$ for $z$ inside the obstacle, while there is no visible change to the domain where $W_{M N}(z)>1$.

Nonscattering fields are displayed in Figure 2(a)-(c). The plots seem to indicate that $\left|v_{g_{M, N, z}}(x)\right|$ depends very little on the position of $z$. Even for $z \in D$, as in Figure 2(b), the value is quite large away from the obstacle, which is more than could be expected from Theorem 3.2.

4. Plane wave scattering for open arcs. At the heart of the theory above is Green's theorem; hence one cannot directly apply the factorization technique described therein to scattering from cracks. Fortunately, the theory can be modified, 
yielding a technique for "imaging" cracks that, for all practical purposes, is identical to the method developed above. We treat the two-dimensional case here and consider weak solutions to the Dirichlet problem

$$
\left(\triangle+k^{2}\right) u=0 \quad \text { in } D_{\Gamma}:=\mathbb{R}^{2} \backslash \Gamma, \quad u=f \quad \text { on } \Gamma,
$$

where $\Gamma \subset \mathbb{R}^{2}$ is a piecewise analytic curve with closed relative interior that has a parameterization of the form $x=z(s), s \in[-1,1]$, for some injective analytic function $z:[-1,1] \rightarrow \mathbb{R}^{2}$ with $z^{\prime}(s) \neq 0$ for all $s$. In addition to (4.1) the scattered field satisfies the Sommerfeld radiation condition (2.2).

For $f \in H^{1 / 2}(\Gamma), u \in H_{\text {loc }}^{1}\left(D_{\Gamma}\right)$ is a weak solution to (4.1) - that is, $u$ also satisfies the Sommerfeld radiation condition and $\int_{D_{\Gamma}}\left(\nabla u \cdot \nabla \bar{\phi}-k^{2} u \bar{\phi}\right) d x=0$ for all compactly supported test functions $\phi \in H^{1}\left(D_{\Gamma}\right)$ with $\left.\phi\right|_{\Gamma}=0$. The far field patterns of these solutions uniquely determine the cracks [19]. We denote the far field operator generated by incident plane waves by $F_{\Gamma}$ and the data-to-pattern operator $G_{\Gamma}: H^{1 / 2}(\Gamma) \rightarrow L^{2}(\mathbb{S})$. We impose the following assumption to guarantee denseness of the far field patterns.

Assumption 4.1. There is no Herglotz wave function that vanishes on $\Gamma$.

TheOREM 4.2. Let Assumption 4.1 hold. The data-to-pattern operator $G_{\Gamma}$ : $H^{1 / 2}(\Gamma) \rightarrow L^{2}(\mathbb{S})$ has the following properties:

1. $G_{\Gamma}$ is a compact, injective, and bounded linear operator with dense range.

2. The ranges of $G_{\Gamma}$ and $\left(F_{\Gamma}^{*} F_{\Gamma}\right)^{1 / 4}$ coincide: $\mathcal{R}\left(G_{\Gamma}\right)=\mathcal{R}\left(\left(F_{\Gamma}^{*} F_{\Gamma}\right)^{1 / 4}\right)$.

3. The operator $\left(F_{\Gamma}^{*} F_{\Gamma}\right)^{-1 / 4} G_{\Gamma}$ is a norm isomorphism from $H^{1 / 2}(\Gamma)$ to $L^{2}(\mathbb{S})$.

4. Let $\widetilde{H}(T)$ denote the completion of $C_{0}^{\infty}(T)$ with respect to $H^{-1 / 2}(C)$, and $C$ be the extension of $T$ to a simple closed curve. For any analytic nonintersecting arc $T$ and density $\rho \in \widetilde{H}^{-1 / 2}(T)$ define

$$
r_{T}(\widehat{x}):=\int_{T} \rho(y) e^{-i k \widehat{x} \cdot y} d s(y), \quad \widehat{x} \in \mathbb{S} .
$$

Then $r_{T}(\widehat{x}) \in \mathcal{R}\left(G_{\Gamma}\right)$ if and only if $T \subset \Gamma$.

Proof. Boundedness and injectivity of $G_{\Gamma}$ are immediate. Compactness of $G_{\Gamma}$ follows by extending the arc $\Gamma$ to an arbitrary simple closed curve and applying the standard argument for compactness of data-to-pattern operators for Dirichlet problems (see, for instance, [17, Lemma 1.13]). Next note that the far field operator has the factorization [18, Lemma 3.4]

$$
F_{\Gamma}=\sqrt{8 \pi k} e^{i \pi / 4} G_{\Gamma} S_{\Gamma}^{*} G_{\Gamma}^{*}
$$

where $S_{\Gamma}$ is the single-layer boundary operator on $\Gamma$. Since $F_{\Gamma}$ is injective, so is $G_{\Gamma}^{*}$ as $S_{\Gamma}^{*}$ is injective, whence point 1 follows. Properties $2-3$ are the content of [18, Theorem 3.7]; property 4 is established by [18, Theorem 3.8].

By the spectral theorem the far field operator $F_{\Gamma}$ admits an eigensystem $\left(\lambda_{n}, \xi_{n}\right)$ such that the eigenfunctions form a complete orthonormal basis of $L^{2}(\mathbb{S})$ and the eigenvalues have a unique cluster point at 0 . For $x, z \in \mathbb{R}^{2}$ let $M, N \in \mathbb{N}$ with $M \leq N$ and define

$$
g_{M, N, T}:=\sum_{n=M}^{N} \frac{\left\langle r_{T}, \xi_{n}\right\rangle}{\left|\lambda_{n}\right|^{1 / 2}} \xi_{n} \quad \text { and } \quad \widehat{g}_{M, N, T}:=\frac{1}{\left\|g_{M, N, T}\right\|} \sum_{n=M}^{N} \frac{\left\langle r_{T}, \xi_{n}\right\rangle}{\left|\lambda_{n}\right|} \xi_{n} .
$$


In order to distinguish different functions $r_{T}$ we center the curve segment on the point $x$ and denote this by $T_{x}$. The definition of $\widehat{g}_{M, N, T}$ yields the following identity:

$$
v_{\widehat{g}_{M, N, T_{x}}}(z):=\int_{\mathbb{S}} \overline{r_{T_{z}}(\widehat{\eta})} \widehat{g}_{M, N, T_{x}}(\widehat{\eta}) d s(\widehat{\eta})=\frac{1}{\left\|g_{M, N, T_{x}}\right\|} \sum_{n=M}^{N} \frac{\left\langle r_{T_{x}}, \xi_{n}\right\rangle}{\left|\lambda_{n}\right|} \overline{\left\langle r_{T_{z}}, \xi_{n}\right\rangle}
$$

By interchanging the order of integration in (4.2), we see that $v_{\widehat{g}_{M, N, T_{x}}}(z)$ is the product of mollified Herglotz wave functions:

$$
v_{\widehat{g}_{M, N, T_{x}}}(z)=\frac{1}{\left\|g_{M, N, T_{x}}\right\|} \sum_{n=M}^{N} \frac{1}{\left|\lambda_{n}\right|}\left\langle\varphi_{x}, v_{\xi_{n}}\right\rangle_{T_{x}} \overline{\left\langle\varphi_{z}, v_{\xi_{n}}\right\rangle_{T_{z}}} .
$$

Here $\varphi_{x}$ is the mollification function centered on $x, v_{\xi_{n}}$ is the Herglotz wave function with density $\xi_{n}$, and $\langle\cdot, \cdot\rangle_{T_{x}}$ is the Euclidean inner product with respect to the seminorm on $T_{x}$. The next result is an analogue of Theorems 3.1 and 3.2.

TheOrem 4.3. Under Assumption 4.1, given any $\epsilon>0, \Gamma_{0} \subset \Gamma$ closed, and $K \subset \mathbb{R}^{2} \backslash \Gamma$ compact, there is a radius $\delta>0$ and $M, N \in \mathbb{N}$ with $M<N$ such that

$$
\begin{array}{ll}
\left|v_{\widehat{g}_{M, N, T_{x}}}(z)\right|<\epsilon & \text { for } z \in \Gamma_{0}, T_{z} \subset \Gamma, x \in \mathbb{R}^{2} ; \\
\left|v_{\widehat{g}_{M, N, T_{x}}}(z)\right|>\frac{1}{\epsilon} & \text { for } x, z \in K \quad \text { with } \quad x \in B_{\delta}(z) .
\end{array}
$$

The proof is very similar to the proofs of Theorems 3.1 and 3.2 , and we omit it here.

As with Dirichlet obstacles and inhomogeneous media we determine the shape and location of the crack by evaluating the magnitude of the constructed incident field $v_{\widehat{g}_{M, N, T_{z}}}(z)$. Comparing Theorem 4.2.4 to Theorem 2.2, we note what appears to be a fundamental difference between the factorization theory for scattering from cracks and scattering from obstacles or inhomogeneous media: in the latter cases the scatterer is found by conducting a pointwise search to determine those points $z$ for which $\Phi^{\infty}(\cdot, z) \in \mathcal{R}(G)$, whereas for cracks one must search along curves $T$. It is common to make the approximation $r_{T}(\cdot) \approx \Phi^{\infty}(\cdot, z)[18,4]$. We will essentially take the same approach; however, we show explicitly how this approximation depends on the sampling rate in the near field of the crack. We next compute an explicit estimate of the approximation of $r_{T_{z}}$ by the far field of a point source centered on $T_{z}$.

Consider the segment of $\Gamma$ with arclength $\delta$ centered at $z$, and the $C_{0}^{\infty}$ mollifying function $\varphi_{z}(y)$ satisfying $\int_{T_{z}} \varphi_{z}(y) d s(y)=1$. Writing $T_{z}$ in terms of the parameterization $y:[-t, t] \rightarrow \mathbb{R}^{2}$ for $t>0$, where $y(0)=z$, we have

$$
r_{T_{z}}(\widehat{\eta})=\int_{-t}^{t} \varphi_{z}(y(s)) e^{-i k \widehat{\eta} \cdot y(s)} y^{\prime}(s) d s=e^{-i k \widehat{\eta} \cdot z} \int_{-t}^{t} \varphi_{z}(w(s)+z) e^{-i k \widehat{\eta} \cdot w(s)} w^{\prime}(s) d s .
$$

Here $w(s)=y(s)-z$, and hence $w(0)=0$. Now expanding $e^{-i k \hat{\eta} \cdot w(s)}$ yields

$r_{T_{z}}(\widehat{\eta})=e^{-i k \widehat{\eta} \cdot z} \int_{-t}^{t} \varphi_{z}(w(s)+z)\left(1-\frac{\left(k \widehat{\eta} \cdot w^{\prime}(0)\right)^{2} s^{2}}{2}+O\left(s^{3}\right)\right) w^{\prime}(s) d s=e^{-i k \hat{\eta} \cdot z}+o(\delta)$.

Since the behavior established by Theorem 4.3 is independent of the segment $T_{z}$ we conclude that the dominant part of the behavior in (4.3) is due to the leadingorder term of $r_{T_{z}}$. In other words, $\sqrt{8 \pi k} e^{-i \pi / 4} \Phi^{\infty}(\widehat{\eta}, z):=e^{-i k \widehat{\eta} \cdot z}$ is a sufficient 


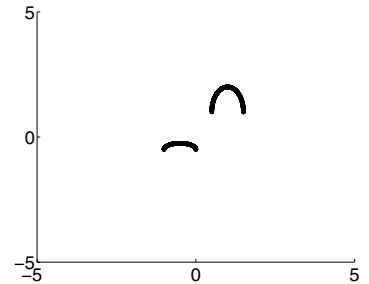

(a)

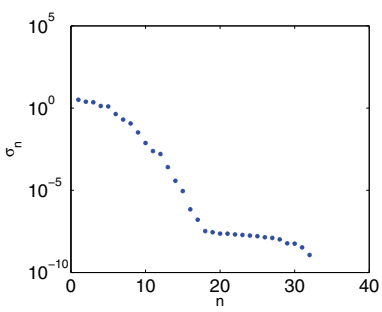

(b)

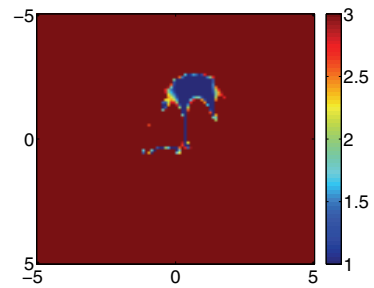

(c)

Fig. 3. (a) Sound-soft arcs to be recovered. (b) Decay of the singular values of the far field operator sampled at 32 observation and 32 incident plane waves with directions evenly distributed on $[-\pi, \pi]$. (c) The magnitude of the incident field $W_{M, N}(z)$ calculated by (4.4), with $M=20$ and $N=32$.

test function for implementation of the MUSIC algorithm. This is demonstrated in Figure 3, where we determine the shape and location of sound-soft cracks by plotting

$$
W_{M, N}(z)=\left|\beta v_{\widehat{g}_{M, N, z}}(z)\right|^{2}=\sum_{n=M}^{N} \frac{1}{\left|\lambda_{n}\right|}\left|\left\langle\Phi^{\infty}(\cdot, z), \xi_{n}\right\rangle\right|^{2} .
$$

5. Using useless data. The data required for the construction of the densities $\hat{g}_{M, N, z}$ is spectral data corresponding to small eigenvalues of the far field operator. In general, due to superalgebraic decay of the eigenvalues of $F$, the size of these eigenvalues will be smaller than the noise level of any physical measurement device or background clutter. Hence, the available data would seem to be useless for implementing nonscattering fields or a MUSIC algorithm for extended scatterers. Surprisingly, this is not the case. We show next that it is not critical which functions are used to construct the indicator function and corresponding nonscattering fields, provided that they are orthogonal to the signal space. In section 6 , we will consider similar questions in a fully discrete setting.

Let us assume that we are given some approximation $F^{\delta}$ of the far field operator $F$ with $\left\|F-F^{\delta}\right\|<\delta\|F\|$, where the norm $\|\cdot\|$ denotes the operator norm in $L^{2}(\mathbb{S})$. The approximation $F^{\delta}$ is typically finite-dimensional. Due to the noise on $F^{\delta}$, in what follows we will work with the singular value decomposition

$$
F^{\delta} \eta=\sum_{j=1}^{N} \sigma_{j}^{\delta}\left\langle\eta, \eta_{j}^{\delta}\right\rangle \xi_{j}^{\delta}, \quad \xi \in L^{2}(\mathbb{S}) .
$$

For $\delta=0$ we find $F=F^{0}$ and denote $\lambda_{j}=s_{j} \sigma_{j}^{0}$ and $\xi_{j}=\xi_{j}^{0}$, where $\left(\lambda_{j}, \xi_{j}\right)$ is an eigensystem of the normal operator $F$ and $s_{j}$ a complex number of magnitude 1 . Working with the singular value decomposition is necessary, as we cannot guarantee normality of the perturbed operators $F^{\delta}$ and thus existence of an orthogonal system of eigenvectors. The singular vectors, however, will always be orthogonal. For $M<N \in \mathbb{N}$ we define the subspaces

$$
U_{M N}^{\delta}=\operatorname{span}\left\{\xi_{M}^{\delta}, \ldots, \xi_{N}^{\delta}\right\}, \quad U_{M \infty}=U_{1 M-1}^{\perp}, \quad \text { and } \quad U_{M \infty}^{\delta}=\left(U_{1 M-1}^{\delta}\right)^{\perp},
$$

and by $P_{M N}^{\delta}$ we denote the orthogonal projection of $L^{2}(\mathbb{S})$ onto $U_{M N}^{\delta}$. Continuity of the spectrum and the eigenspaces of a bounded linear operator with respect to bounded perturbations yields bounds on the difference $P_{1 N}^{\delta}-P_{1 N}$. 
Lemma 5.1. Let $N \in \mathbb{N}$ and $\delta>0$, and let $N^{*}$ be the largest integer with $\lambda_{N^{*}}=\lambda_{N}$. We assume that $\left.|| \lambda_{N^{*}}\right|^{2}-\left|\lambda_{N^{*}+1}\right|^{2} \mid>C(F, \delta):=4\|F\|^{2} \delta+2\|F\|^{2} \delta^{2}$. Then

$$
\left\|P_{1 N}^{\delta}-P_{1 N}\right\| \leq \frac{C(F, \delta) N^{*}}{\left.|| \lambda_{N^{*}}\right|^{2}-\left|\lambda_{N^{*}+1}\right|^{2} \mid-C(F, \delta)} .
$$

Proof. For the proof of this lemma, we assume that the reader is familiar with basic definitions from spectral theory. The estimate follows from perturbation theory of linear operators combined with the fact that $F^{*} F \xi_{j}=\left|\lambda_{j}\right|^{2} \xi_{j}$. Therefore the projections $P_{M N}$ can be expressed as projections onto eigenspaces of $F^{*} F$. Note that $\left\|F^{\delta *} F^{\delta}-F^{*} F\right\| \leq 2\|F\|^{2} \delta+\|F\|^{2} \delta^{2}$. We can represent the orthogonal projection on the eigenspaces corresponding to the first $N^{*}$ eigenvalues of $F^{*} F$ by a contour integral (see, e.g., [21, section 4]): we integrate the resolvent $\lambda \mapsto\left(\lambda I-F^{*} F\right)^{-1}$ over a positively oriented path $\gamma$ in the complex plane, which encloses exactly the first $N^{*}$ eigenvalues. More precisely, the index of the first $N^{*}$ eigenvalues of $F^{*} F$ with respect to $\gamma$ is one, while the index of all other eigenvalues vanishes. Then

$$
P_{1 N}=\frac{1}{2 \pi i} \int_{\gamma}\left(\lambda I-F^{*} F\right)^{-1} d \lambda
$$

and if $\delta>0$ is small enough such that $\gamma$ does not meet the spectrum of $F^{\delta *} F^{\delta}$, the analogous formula holds for $P_{1 N}^{\delta}$ with $F^{*} F$ replaced by $F^{\delta *} F^{\delta}$. Therefore the resolvent identity

$$
\left(\lambda I-F^{*} F\right)^{-1}-\left(\lambda I-F^{\delta *} F^{\delta}\right)^{-1}=\left(\lambda I-F^{*} F\right)^{-1}\left(F^{*} F-F^{\delta *} F\right)\left(\lambda I-F^{\delta *} F^{\delta}\right)^{-1},
$$

for $\lambda$ in the resolvent set of $F^{*} F$ and $F^{\delta *} F^{\delta}$, and the estimate $\left\|\left(\lambda I-F^{*} F\right)^{-1}\right\|_{L^{2}(\mathbb{S})} \leq$ $\left.\sup _{j \in \mathbb{N}}|| \lambda_{j}\right|^{2}-\left.\lambda\right|^{-1}$ from [13, V.4.3] imply

$$
\left\|P_{1 N}^{\delta}-P_{1 N}\right\|_{L^{2}(\mathbb{S})} \leq\left(2\|F\|^{2} \delta+\delta^{2}\|F\|^{2}\right) \frac{|\gamma|}{2 \pi} \sup _{\lambda \in \gamma}\left[\left.\sup _{j \in \mathbb{N}}|| \lambda_{j}\right|^{2}-\left.\left.\lambda\right|^{-1} \sup _{j \in \mathbb{N}}|| \lambda_{j}^{\delta}\right|^{2}-\left.\lambda\right|^{-1}\right] .
$$

Here, $|\gamma|$ means the length of the curve $\gamma$. We need to specify this contour. Consider a ball in the complex plane of radius $\left.|| \lambda_{N}\right|^{2}-\left|\lambda_{N+1}\right|^{2} \mid / 2$ around each of the points $\left|\lambda_{j}\right|^{2}, j \leq N^{*}$. These balls might overlap, and we choose the piecewise smooth curve $\gamma$ to be the boundary of the union of these balls. For $\lambda \in \gamma$ we have by construction $\left.|| \lambda_{j}\right|^{2}-\lambda|=|\left|\lambda_{N}^{*}\right|^{2}-\left|\lambda_{N^{*}+1}\right|^{2} \mid / 2$, and continuous dependence of the spectrum on its operator [21, Theorem 4.1] gives $\left.|| \lambda_{j}^{\delta}\right|^{2}-\lambda|\geq|\left|\lambda_{N}^{*}\right|^{2}-\left|\lambda_{N^{*}+1}\right|^{2} \mid / 2-2\|F\|^{2} \delta-\|F\|^{2} \delta^{2}$. Since $|\gamma|$ can be estimated from above by $\pi\left(\left.|| \lambda_{N}^{*}\right|^{2}-\left|\lambda_{N^{*}+1}\right|^{2} \mid\right) N^{*}$, we find

$$
\left\|P_{1 N}^{\delta}-P_{1 N}\right\|_{L^{2}(\mathbb{S})} \leq \frac{\left(2\|F\|^{2} \delta+\|F\|^{2} \delta^{2}\right) N^{*}}{\left\|\left.\lambda_{N}^{*}\right|^{2}-\left|\lambda_{N^{*}+1}\right|^{2} \mid / 2-2\right\| F\left\|^{2} \delta-\right\| F \|^{2} \delta^{2}} .
$$

For the rest of this section we investigate the perturbed indicator

$$
W_{M N}^{\delta}(z):=\sum_{n=M}^{N} \frac{1}{\left|\sigma_{n}^{\delta}\right|}\left|\left\langle\Phi^{\infty}(\cdot, z), \xi_{n}^{\delta}\right\rangle\right|^{2}, \quad z \in \mathbb{R}^{m} .
$$

This function is the analogue of (3.6) using the singular values $\sigma_{n}^{\delta}$ of the perturbed far field operator $F^{\delta}$, since we cannot guarantee normality of $F^{\delta}$ and thus existence of 


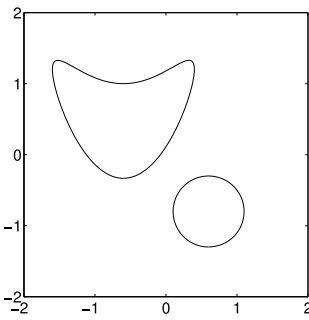

(a)

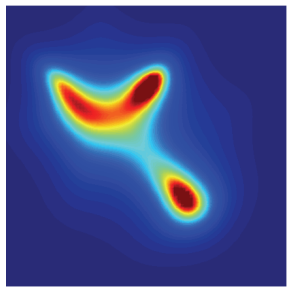

(d)

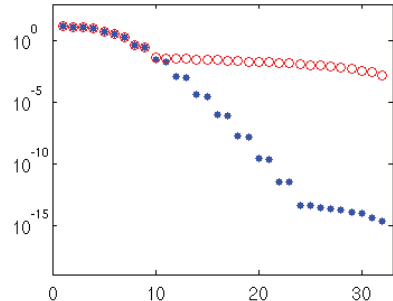

(b)

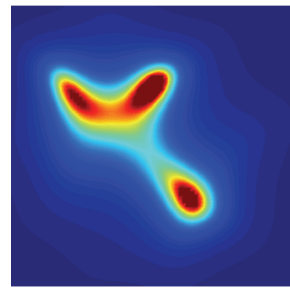

(e)

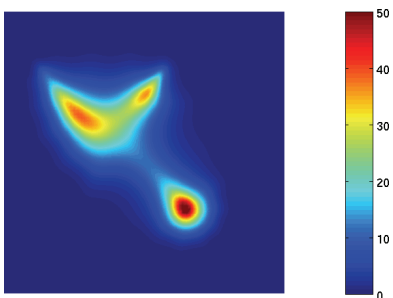

(c)

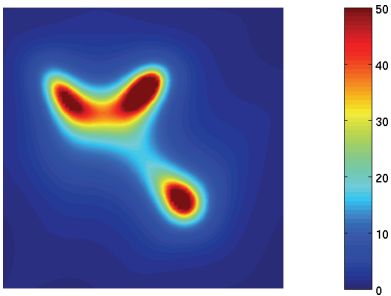

(f)

FIG. 4. Inverse medium scattering. (a) Location of the penetrable scatterers. (b) Singular values of $F_{\mathrm{FD}}$ and $F_{\mathrm{FD}}^{0.01}$ generated from 32 incident plane waves with directions uniformly distributed on $[-\pi, \pi]$. (c)-(f) Plot of $W_{520}(z)^{-1}$ for noise levels (c) $\delta=0,(\mathrm{~d}) \delta=0.01$, (e) $\delta=0.05$, and (f) $\delta=0.1$

an orthogonal system of eigenvectors. We will assume as a notational simplification that all singular values have multiplicity one, as the extension to multiple singular values is immediate. Crucial for our analysis is the following estimate for $P_{M \infty}^{\delta}$ : Due to Lemma 5.1 we have

$$
\begin{aligned}
& \left\|P_{M \infty}^{\delta}-P_{M \infty}\right\|=\left\|I-P_{1 M-1}^{\delta}+\left(I-P_{1 M-1}\right)\right\| \\
& \quad=\left\|P_{1 M-1}^{\delta}-P_{1 M-1}\right\| \leq \frac{C(F, \delta) M}{\left.|| \lambda_{M-1}\right|^{2}-\left|\lambda_{M}\right|^{2} \mid-C(F, \delta)} .
\end{aligned}
$$

Consider a point $z \in D$. For such a point we can estimate

$$
\begin{aligned}
W_{M N}^{\delta}(z) & \leq \frac{1}{\min _{j=M, \ldots, N}\left|\sigma_{j}^{\delta}\right|} \sum_{j=M}^{N}\left|\left\langle\Phi^{\infty}(\cdot, z), \xi_{j}^{\delta}\right\rangle_{N}\right|^{2} \leq \frac{1}{\min _{j=M, \ldots, N}\left|\sigma_{j}^{\delta}\right|}\left\|P_{M \infty}^{\delta} \Phi^{\infty}(\cdot, z)\right\|^{2} \\
& \leq \frac{1}{\min _{j=M, \ldots, N}\left|\sigma_{j}^{\delta}\right|}\left(\left\|P_{M \infty} \Phi^{\infty}(\cdot, z)\right\|^{2}+\frac{C(F, \delta) M\left\|\Phi^{\infty}(\cdot, z)\right\|_{L^{2}(\mathbb{S})}^{2}}{\left.|| \lambda_{M-1}\right|^{2}-\left|\lambda_{M}\right|^{2} \mid-C(F, \delta)}\right) \\
& \leq \frac{\max _{j=M, \ldots, N}\left|\lambda_{j}\right|}{\min _{j=M, \ldots, N}\left|\sigma_{j}^{\delta}\right|}\left(\sum_{j=M}^{N} \frac{\left|\left\langle\Phi^{\infty}(\cdot, z), \xi_{j}\right\rangle_{N}\right|^{2}}{\left|\lambda_{j}\right|}+\frac{C(F, \delta) M\left\|\Phi^{\infty}(\cdot, z)\right\|_{L^{2}(\mathbb{S})}^{2}}{\left.|| \lambda_{M-1}\right|^{2}-\left|\lambda_{M}\right|^{2} \mid-C(F, \delta)}\right) \\
(5.2) \quad & \leq \frac{\max _{j=M, \ldots, N}\left|\lambda_{j}\right|}{\min _{j=M, \ldots, N}\left|\sigma_{j}^{\delta}\right|}\left(W_{M N}(z)+\frac{C(F, \delta) M\left\|\Phi^{\infty}(\cdot, z)\right\|_{L^{2}(\mathbb{S})}^{2}}{\left.|| \lambda_{M-1}\right|^{2}-\left|\lambda_{M}\right|^{2} \mid-C(F, \delta)}\right) .
\end{aligned}
$$

We will discuss this estimate in the context of a numerical example. The problem is an inverse medium scattering problem. The configuration is shown in Figure 4(a). 
Inside the circle and the kite-shaped region, the index of refraction is 10 , while it is 1 outside these areas; a constant contrast is, of course, no requirement of the inversion method, as we showed in section 3 . The wavenumber is $k=2$. We use 32 incident plane waves uniformly distributed on $[-\pi, \pi]$ to generate the fully discrete far field operator, $F_{\mathrm{FD}}$. The spectral norm of $F_{\mathrm{FD}}$ is approximately 3.54 in this case.

The singular values of the corresponding far field operator are shown in Figure 4(b). The following table displays maximum values of $\delta$ such that the assumption $\left.|| \lambda_{M-1}\right|^{2}-\left|\lambda_{M}\right|^{2} \mid>C(F, \delta)$ is valid for given $M$.

\begin{tabular}{ccccccc}
\hline$M$ & 2 & 3 & 4 & 5 & 6 & 7 \\
\hline$\delta$ & 0.5132 & 0.5611 & 0.2320 & 0.2498 & 0.0629 & 0.0222 \\
\hline
\end{tabular}

These values show that the estimate can be be applied for, e.g., $\delta=0.1$ up to $M=5$ for this example. In cases where it can be applied, estimate (5.2) guarantees stability of the MUSIC algorithm with respect to localizing the scatterer. The perturbed indicator function $W_{M N}^{\delta}$ cannot be arbitrarily larger than $W_{M N}$. Note that all quantities in the estimate except for $W_{M N}$ itself are independent of $z$.

Stability of the MUSIC algorithm under noise can also be clearly seen in the numerical example. Figure 4(c) is a plot of the reciprocal value of $W_{520}$ for unperturbed data, while Figures 4(d)-(f) show reconstruction for various levels of artificial noise added to $F_{\mathrm{FD}}$. Here, perturbed data is obtained by adding uniformly distributed random numbers to the entries of the matrix $F_{\mathrm{FD}}$.

For points outside the scatterer we are not able to provide such a complete analysis for noisy data. Obviously, $W_{M N}^{\delta}(z) \geq\left(\max _{j=M, \ldots, N}\left|\sigma_{j}^{\delta}\right|\right)^{-1}\left\|P_{M N}^{\delta} \Phi^{\infty}(\cdot, z)\right\|^{2}$, but it is unclear to us how to reasonably estimate the latter quantity from below. Indeed, our numerical experiments in Figure 4 indicate that $W_{M N}^{\delta}(z)$ can be rather small in a neighborhood outside $D$.

6. The factorization method with noisy data. Estimates similar to those presented in section 5 can be derived for the factorization method in the case of noisy data. How many terms to use in the Picard series for reconstructions of obstacles and inclusions has been a long-standing issue. The methods derived in this paper provide a partial answer. In contrast to the analysis above, we make explicit use of the finite dimensionality present in any numerical computation.

As an example we again consider scattering from the sound-soft obstacle shown in Figure 1(a) with $k=1$. We use the same 25 incident plane waves as in Figure 1. As noise will be introduced, we make use of the singular value decomposition of $F_{\mathrm{FD}}$,

$$
F_{\mathrm{FD}} \xi=\sum_{j=1}^{N} \sigma_{j}\left(\xi \cdot \xi^{(j)}\right) \eta^{(j)}, \quad \xi \in \mathbb{C}^{N}
$$

with the normalized scalar product $\xi \cdot \eta=(2 \pi / N) \sum_{j=1}^{N} \xi_{j} \overline{\eta_{j}}$ for $\xi, \eta \in \mathbb{C}^{N}$. For simplicity we have taken $J$, the number of incident plane waves used to generate the fully discrete operator, to be the same as the number of terms in the Picard series, $N$. Using the normalization means that the scalar product is exactly the composite rectangular rule applied to the $L^{2}$-scalar product for the corresponding interpolating functions in $L^{2}(\mathbb{S})$.

We generate a reconstruction of the obstacle, by introducing the vector $\phi_{z}=$ 


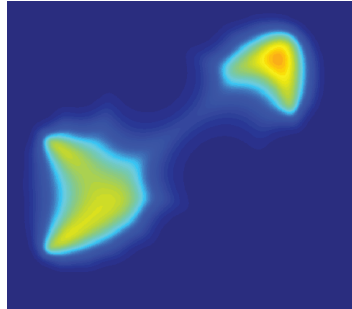

(a) singular values 1-25 (correct)

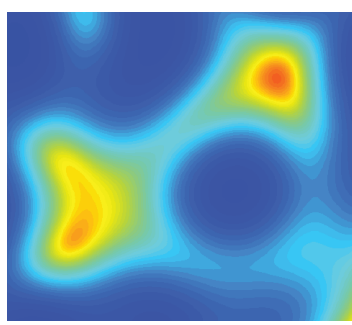

(d) singular values $1-10$ (noisy)

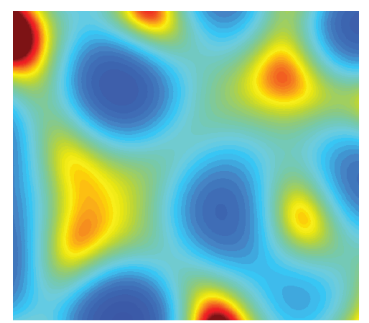

(b) singular values 1-8 (noisy)

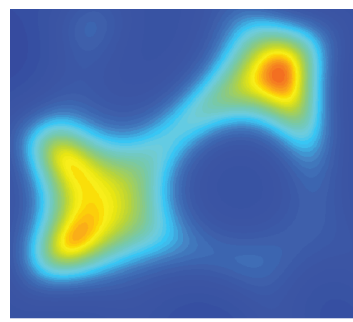

(e) singular values $1-15$ (noisy)

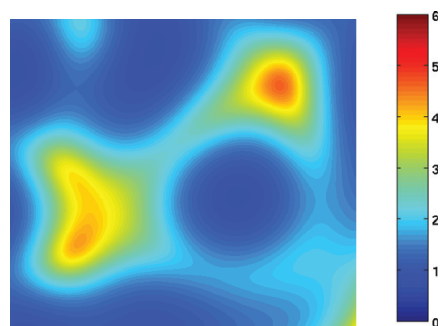

(c) singular values 1-9 (noisy)

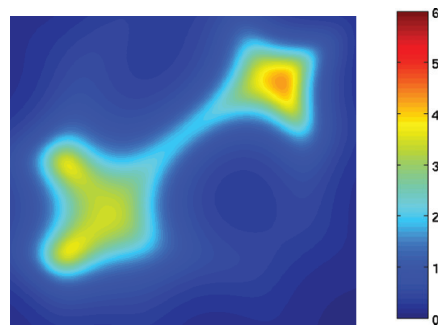

(f) singular values 1-25 (noisy)

FIG. 5. Reconstructions using noisy singular values.
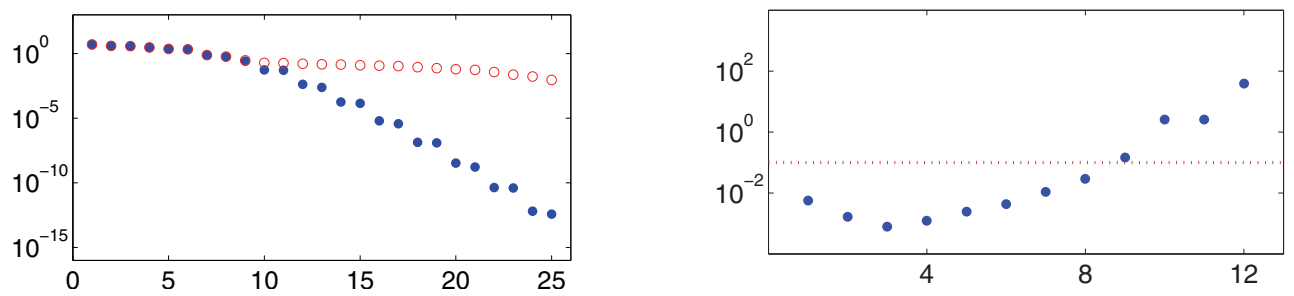

FIG. 6. Singular values of the correct and perturbed discrete operators (left) and the relative error in singular values 1-12 (right). The dotted line indicates a relative error of 0.1 .

$\left(\Phi^{\infty}\left(\hat{x}_{0}, z\right), \ldots, \Phi^{\infty}\left(\hat{x}_{N-1}, z\right)\right)^{\top}$ and defining the function

$$
W_{M}(z):=\sum_{j=1}^{M} \frac{\left|\phi_{z} \cdot \xi^{(j)}\right|^{2}}{\left|\sigma_{j}\right|} .
$$

This is the discrete analogue of (3.6). As with the perturbed far field operator given by (5.1), we cannot assume the existence of eigenvalues; hence we formulate the indicator function in terms of the singular values $\sigma_{j}$. The reciprocal value of $W_{M}$ is plotted on a grid for $M=25$, yielding Figure 5(a).

Next, we add noise to the data. By adding uniformly distributed random numbers to all entries of $F_{\mathrm{FD}}$, we obtain a perturbed matrix $F_{\mathrm{FD}}^{\delta}$ such that $\left\|F_{\mathrm{FD}}-F_{\mathrm{FD}}^{\delta}\right\|_{2}=$ $\delta\left\|F_{\mathrm{FD}}\right\|_{2}$, where $\|\cdot\|_{2}$ indicates the spectral norm. For an example using $\delta=0.05$, the singular values of $F_{\mathrm{FD}}^{\delta}$ are shown in Figure 6(left) as circles, while the correct singular values are shown as crosses. The plot on the right shows the relative error in the first 12 singular values of $F_{\mathrm{FD}}^{\delta}$. 
Using only those singular values with a relative error of less than $10 \%$, i.e., the first eight, gives Figure 5(b). Figures 5(c)-(f) show reconstructions using more and more of the singular values of $F_{\mathrm{FD}}^{\delta}$ with a relative error of more than $10 \%$. While the values computed for points inside the obstacles do not change appreciably, the reconstruction significantly improves for points outside the obstacle. It appears that using incorrect spectral information is not only helpful but crucial for a good reconstruction using the factorization method. We will provide some insight into why this is the case.

The problem with Figure 5(b) is that, even though $W_{M}(z) \rightarrow \infty(M \rightarrow \infty)$ for $z \notin D$, the values of $W_{M}(z)$ computed for some points $z$ outside the obstacle are still rather small. The divergence of the Picard series for such points influences the behavior of $W_{M}(z)$ for larger values of $M$ only.

We note that for any continuous function $g$ on $\mathbb{S}$ and $\xi \in \mathbb{C}^{N}$ such that $\xi_{j}=g\left(\hat{x}_{j}\right)$,

$$
\phi_{z} \cdot \xi \approx\left\langle\Phi^{\infty}(\cdot, z), g\right\rangle=\beta \int_{S} e^{-\mathrm{i} k z \cdot \widehat{\eta}} \overline{g(\widehat{\eta})} d s(\widehat{\eta})=\beta \overline{v_{g}(z)}
$$

A general result on Herglotz functions is the asymptotic formula

$$
v_{g}(z)=\gamma g(\hat{z}) \frac{e^{\mathrm{i} k|z|}}{|z|^{\frac{m-1}{2}}}-\gamma g(-\hat{z}) \frac{e^{-\mathrm{i} k|z|}}{|z|^{\frac{m-1}{2}}}+\mathrm{o}\left(\frac{1}{|z|}\right), \quad|z| \rightarrow \infty
$$

which is well known in the literature (see, e.g., [10]). The proof can be carried out by expanding $v_{g}$ in spherical harmonics in angular and Bessel functions in radial directions. This gives the asymptotic behavior with certain far field patterns. The exact patterns can be obtained by computing the coefficients in the expansion explicitly using the Funk-Hecke formula. As a consequence, any partial sum of the Picard series will be arbitrarily small on an appropriately chosen subset of the plane. This is not a contradiction to the factorization method, which states that, given any compact set away from the obstacle, an appropriately chosen partial sum of the Picard series will be arbitrarily large.

Next, we provide a partial explanation for why it is beneficial to use incorrect spectral information for the reconstruction. Suppose that the first $M$ singular values and corresponding vectors are known exactly, while the singular triplets $\left(\sigma_{j}, \xi^{(j)}, \eta^{(j)}\right)$ are replaced by $\left(\sigma_{j}^{\delta}, \xi^{(j, \delta)}, \eta^{(j, \delta)}\right), j=M+1, \ldots, N$. Note, however, that these form a singular system of the matrix $F_{\mathrm{FD}}^{\delta}$, and hence the singular vectors form an orthonormal basis of $\mathbb{C}^{N}$.

Next we define two subspaces, $U_{M}=\operatorname{span}\left\{\xi^{(1)}, \ldots, \xi^{(M)}\right\}$ and $V_{M}=U_{M}^{\top}$. From the properties of the singular value decomposition, we immediately obtain

$$
V_{M}=\operatorname{span}\left\{\xi^{(M+1)}, \ldots, \xi^{(N)}\right\}=\operatorname{span}\left\{\xi^{(M+1, \delta)}, \ldots, \xi^{(N, \delta)}\right\}
$$

We denote the orthogonal projections onto these subspaces by $P_{M}: \mathbb{C}^{N} \rightarrow U_{M}$ and $Q_{M}: \mathbb{C}^{N} \rightarrow V_{M}$, respectively. Finally, we define the perturbed indicator

$$
W_{N}^{\delta}(z)=W_{M}(z)+\sum_{j=M+1}^{N} \frac{\left|\phi_{z} \cdot \xi^{(j, \delta)}\right|^{2}}{\left|\sigma_{j}^{\delta}\right|} .
$$


Consider now a point $z \in D$. For such a point we can estimate

$$
\begin{aligned}
W_{N}^{\delta}(z)-W_{M}(z) & \leq \frac{1}{\min _{j=M+1, \ldots, N}\left|\sigma_{j}^{\delta}\right|} \sum_{j=M+1}^{N}\left|\phi_{z} \cdot \xi^{(j, \delta)}\right|^{2} \\
& =\frac{1}{\min _{j=M+1, \ldots, N}\left|\sigma_{j}^{\delta}\right|}\left\|Q_{M} \phi_{z}\right\|^{2} \leq \frac{\max _{j=M+1, \ldots, N}\left|\sigma_{j}\right|}{\min _{j=M+1, \ldots, N}\left|\sigma_{j}^{\delta}\right|} \sum_{j=M+1}^{N} \frac{\left|\phi_{z} \cdot \xi^{(j)}\right|^{2}}{\left|\sigma_{j}\right|} \\
& \leq W_{N}(z)-W_{M}(z)+\left(\frac{\max _{j=M+1, \ldots, N}\left|\sigma_{j}\right|}{\min _{j=M+1, \ldots, N}\left|\sigma_{j}^{\delta}\right|}-1\right) \sum_{j=M+1}^{N} \frac{\left|\phi_{z} \cdot \xi^{(j)}\right|^{2}}{\left|\sigma_{j}\right|}
\end{aligned}
$$

This estimate can be interpreted in the following way: at a point inside the obstacle, if using only the correct singular values yields an estimate that is less than the value of the Picard series, then the effect of adding the incorrect terms will not be significant. First, the remainder term $\sum_{j=M+1}^{N}\left|\phi_{z} \cdot \xi^{(j)}\right|^{2} /\left|\sigma_{j}\right|$ will be small for such points. Second, all perturbed singular values are more or less the same size. The effect is most clearly visible in Figures 5(c) and (d), where the values computed for points inside the obstacle decrease significantly. If 15 singular values are used, the ratio $\max _{j=M+1, \ldots, N}\left\{\left|\sigma_{j}\right|\right\} / \min _{j=M+1, \ldots, N}\left\{\left|\sigma_{j}^{\delta}\right|\right\}$ is roughly 2 ; for 25 singular values it is roughly 29 .

Next, we consider the situation for $z \notin D$. As already described in section 5, it is not possible to present a complete analysis with precise estimates in this case. However, useful partial results are possible.

Assume that there is $z \notin D$ such that $\left\|P_{M} \phi_{z}\right\|<\varepsilon\left\|\phi_{z}\right\|$. Consequently,

$$
W_{M}(z)=\sum_{j=1}^{M} \frac{\left|\phi_{z} \cdot \xi^{(j)}\right|^{2}}{\sigma_{j}}<\frac{1}{\min _{j=1, \ldots, M} \sigma_{j}} \sum_{j=1}^{M}\left|\phi_{z} \cdot \xi^{(j)}\right|^{2}<\frac{\varepsilon^{2}\left\|\phi_{z}\right\|^{2}}{\min _{j=1, \ldots, M} \sigma_{j}} .
$$

If $\varepsilon$ is small enough, the behavior of $W_{M}$ indicates (incorrectly) that this point is inside the obstacle. However,

$$
W_{N}^{\delta}(z)-W_{M}(z)=\sum_{j=M+1}^{N} \frac{\left|\phi_{z} \cdot \xi^{(j, \delta)}\right|^{2}}{\sigma_{j}} \geq \frac{\left\|Q_{M} \phi_{z}\right\|^{2}}{\max _{j=M+1, \ldots, N} \sigma_{j}^{\delta}}=\frac{\left(1-\varepsilon^{2}\right)\left\|\phi_{z}\right\|^{2}}{\max _{j=M+1, \ldots, N} \sigma_{j}^{\delta}} .
$$

Noting that $\left\|\phi_{z}\right\|^{2}=(2 \pi / N) \sum_{j=1}^{N}\left|\Phi^{\infty}\left(\hat{x}_{j}, z\right)\right|^{2}=2 \pi|\beta|^{2}$, all quantities in this lower bound except for $\varepsilon$ are independent of $z$. Consequently, using incorrect data will, for example, guarantee that

$$
W_{M}^{\delta}(z)>\frac{3}{4} \frac{2 \pi|\beta|^{2}}{\min _{j=M+1, \ldots, N} \sigma_{j}^{\delta}}
$$

for all $z \notin D$ satisfying $\left\|P_{M} \phi_{z}\right\|<\left\|\phi_{z}\right\| / 2$.

Unfortunately, in the numerical example presented in this section, $\varepsilon>1 / 2$ for all points on the grid. For the point $z=(2.8,-0.3)^{\top}$, where using only the first 8 singular values gives a bad result, the optimal $\varepsilon$ is close to 0.9 . We can predict some increase in the value of $W_{N}^{\delta}(z)$ as opposed to $W_{M}(z)$, but the prediction is far from sharp. 
In conclusion, we have shown that using incorrect spectral data for points inside the scatterer is not too harmful, while the same incorrect spectral data can enhance the contrast on the outside of the scatterer.

Appendix. Discrete approximation of $\boldsymbol{F}$. In this appendix, we study, in two dimensions, the relation of the spectral information of the fully discrete operator $F_{\mathrm{FD}}$ introduced in section 2 to the continuous operator $F$. Although it is plausible that these are closely related, to the authors' knowledge an analysis of this fact has not been published to date. Some related considerations for an inverse elliptic boundary value problem are contained in [21].

We introduce the trigonometric monomials on $L^{2}(S)$ by setting

$$
t_{k}(\hat{x})=\frac{1}{\sqrt{2 \pi}} e^{\mathrm{i} k \varphi}, \quad k \in \mathbb{Z}, \quad \text { where } \quad \hat{x}=\left(\begin{array}{c}
\cos \varphi \\
\sin \varphi
\end{array}\right), \quad \varphi \in[0,2 \pi) .
$$

Note that $\left\{t_{k}: k \in \mathbb{Z}\right\}$ forms a complete orthonormal system of $L^{2}(S)$ and more generally provides a basis of the Sobolev space $H^{s}(S), s \geq 0$, defined by

$$
H^{s}(S)=\left\{g=\sum_{k \in \mathbb{Z}} \alpha_{k} t_{k}: \sum_{k \in \mathbb{Z}}\left(1+k^{2}\right)^{s}\left|\alpha_{k}\right|^{2}<\infty\right\}, \quad\|g\|_{H^{s}}^{2}=\sum_{k \in \mathbb{Z}}\left(1+k^{2}\right)^{s}\left|\alpha_{k}\right|^{2} .
$$

As above, for simplicity we take the number of incident plane waves $J$ used in the construction of $F_{\mathrm{FD}}$ to be the same as the number of terms retained in the Picard series. We introduce the finite-dimensional spaces

$$
T_{2 N-1}=\left\{\sum_{k=-N}^{N} \alpha_{k} t_{k}, \alpha_{k} \in \mathbb{C}\right\}, \quad T_{2 N}=\left\{\sum_{k=-N}^{N} \alpha_{k} t_{k}, \alpha_{k} \in \mathbb{C}, \alpha_{-N}=\alpha_{N}\right\},
$$

for $N \in \mathbb{N}$. Two projection operators on these spaces come into play. By $P_{N}$ : $L^{2}(S) \rightarrow T_{N}$ we denote the orthogonal projection onto $T_{N}$ with respect to the $L^{2}$ scalar product. By $L_{N}: H^{s}(S) \rightarrow T_{N}$ for $s>1 / 2$ we denote the operator of interpolation by a trigonometric polynomial in the points $\left\{\hat{x}_{j}: j=0, \ldots, N-1\right\}$. We remind the reader that functions in $H^{s}(S)$ are continuous if $s>1 / 2$, and hence the interpolation operator makes sense.

We next introduce a semidiscrete operator $F_{\mathrm{SD}}: L^{2}(S) \rightarrow L^{2}(S)$ defined by

$$
F_{\mathrm{SD}} g=L_{N}\left(\frac{2 \pi}{N} \sum_{k=0}^{N-1} u^{\infty}\left(\cdot, \hat{x}_{k}\right)\left(P_{N} g\right)\left(\hat{x}_{k}\right)\right), \quad g \in L^{2}(S) .
$$

As the far field $u^{\infty}$ is analytic with respect to both arguments, we certainly are dealing with a bounded linear operator.

Lemma A.1. Suppose $\lambda \in \mathbb{C} \backslash\{0\}$. Then $\lambda$ is an eigenvalue of $F_{\mathrm{SD}}$ if and only if it is an eigenvalue of $F_{\mathrm{FD}}$.

Proof. Suppose first that $(\lambda, g) \in \mathbb{C} \times L^{2}(S)$ is an eigenpair of $F_{\mathrm{SD}}$. Then $g \in T_{N}$, and we can define $\tilde{g} \in \mathbb{C}^{N}$ by setting $\tilde{g}_{k}=g\left(\hat{x}_{k}\right), k=0, \ldots, N-1$. Noting $P_{N} g=g$ and the interpolation property, an easy computation shows

$$
\left(F_{\mathrm{FD}} \tilde{g}\right)_{j}=\frac{2 \pi}{N} \sum_{k=0}^{N-1} u^{\infty}\left(\hat{x}_{j}, \hat{x}_{k}\right) g\left(\hat{x}_{k}\right)=\left(F_{\mathrm{SD}} g\right)\left(\hat{x}_{j}\right)=\lambda g\left(\hat{x}_{j}\right)=\lambda \tilde{g}_{j} .
$$

Hence $(\lambda, \tilde{g}) \in \mathbb{C} \times \mathbb{C}^{N}$ is an eigenpair of $F_{\mathrm{FD}}$. 
Conversely, suppose that $(\lambda, \tilde{g}) \in \mathbb{C} \times \mathbb{C}^{N}$ is an eigenpair of $F_{\mathrm{FD}}$ with $\lambda \neq 0$. Define $g \in T_{N}$ by $g=(1 / \lambda) L_{N}\left((2 \pi / N) \sum_{k=0}^{N-1} u^{\infty}\left(\cdot, \hat{x}_{k}\right) \tilde{g}_{k}\right)$. Then

$$
\left(P_{N} g\right)\left(\hat{x}_{j}\right)=g\left(\hat{x}_{j}\right)=\frac{1}{\lambda} \frac{2 \pi}{N} \sum_{k=0}^{N-1} u^{\infty}\left(\hat{x}_{j}, \hat{x}_{k}\right) \tilde{g}_{k}=\frac{1}{\lambda}\left(F_{\mathrm{FD}} \tilde{g}\right)_{j}=\tilde{g}_{j},
$$

and we obtain $\lambda g=F_{\mathrm{SD}} g$. This completes the proof.

By next proving that $F_{\mathrm{SD}}$ approximates $F$, we can use results from operator perturbation theory [13] to argue that the first few eigenvalues and eigenspaces of $F_{\mathrm{SD}}$ and $F$ and hence of $F_{\mathrm{FD}}$ and $F$ must be close.

TheOREM A.2. There holds $F_{\mathrm{SD}} \rightarrow F(N \rightarrow \infty)$ in the operator norm on $L^{2}(S)$.

Proof. We first introduce the auxiliary operator $F_{\text {aux }}: L^{2}(S) \rightarrow L^{2}(S)$ by

$$
F_{\text {aux }} g(\hat{x})=\frac{2 \pi}{N} \sum_{k=0}^{N-1} u^{\infty}\left(\hat{x}, \hat{x}_{k}\right)\left(P_{N} g\right)\left(\hat{x}_{k}\right), \quad \hat{x} \in S .
$$

Then, noting $F_{\text {aux }} P_{N}=F_{\text {aux }}$, we have $F-F_{\mathrm{SD}}=F\left(I-P_{N}\right)+\left(F-F_{\text {aux }}\right) P_{N}+$ $F_{\text {aux }}-F_{\mathrm{SD}}$. However, the composite rectangular rule on $S$ integrates trigonometric polynomials of degree at most $N / 2$ exactly, so $\left.\left(F-F_{\text {aux }}\right)\right|_{T_{N}}=0$. Noting further that $F_{\mathrm{SD}}=L_{N} F_{\text {aux }}$, we obtain $F-F_{\mathrm{SD}}=F\left(I-P_{N}\right)+\left(I-L_{N}\right) F_{\text {aux }}$.

We start by estimating the second difference. From [20, Theorem 11.8] we obtain

$$
\left\|\left(I-L_{N}\right) g\right\|_{L^{2}} \leq \frac{C(s)}{N^{s}}\|g\|_{H^{s}}, \quad g \in H^{s}(S),
$$

for any $s>1 / 2$. Furthermore, using the Cauchy-Schwarz inequality,

$$
\begin{aligned}
\left\|F_{\text {aux }} g\right\|_{H^{s}}^{2} & =\frac{4 \pi^{2}}{N^{2}} \sum_{j \in \mathbb{Z}^{2}}\left(1+j^{2}\right)^{s}\left|\sum_{k=0}^{N-1} \int_{S} u^{\infty}\left(\hat{x}, \hat{x}_{k}\right) \overline{t_{j}(\hat{x})} d s(\hat{x})\left(P_{N} g\right)(\hat{x})_{k}\right|^{2} \\
\leq & \frac{4 \pi^{2}}{N^{2}}\left(\sum_{k=0}^{N-1}\left|\left(P_{N} g\right)(\hat{x})_{k}\right|^{2}\right)\left(\sum_{j \in \mathbb{Z}^{2}}\left(1+j^{2}\right)^{s}\left|\sum_{k=0}^{N-1} \int_{S} u^{\infty}\left(\hat{x}, \hat{x}_{k}\right) \overline{t_{j}(\hat{x})} d s(\hat{x})\right|^{2}\right) .
\end{aligned}
$$

As $u^{\infty}$ is analytic, the last factor is bounded independently of $N$ for any $s \geq 0$. Hence

$$
\left\|\left(I-L_{N}\right) F_{\text {aux }} g\right\|_{L^{2}} \leq \frac{C(s)}{N^{s+1}}\left(\sum_{k=0}^{N-1}\left|\left(P_{N} g\right)(\hat{x})_{k}\right|^{2}\right)^{1 / 2}, \quad g \in L^{2}(S) .
$$

From the error estimate for the composite rectangular rule, we obtain

$$
\begin{aligned}
\frac{1}{N} \sum_{k=0}^{N-1}\left|\left(P_{N} g\right)(\hat{x})_{k}\right|^{2} \leq C\left(\left\|P_{N} g\right\|_{L^{2}}^{2}\right. & \left.\frac{\left\|\left(\left|P_{N} g\right|^{2}\right)^{\prime}\right\|_{\infty}}{N}\right) \\
& \leq C\left(\left\|P_{N} g\right\|_{L^{2}}^{2}+\frac{\left\|P_{N} g\right\|_{\infty}\left\|\left(P_{N} g\right)^{\prime}\right\|_{\infty}}{N}\right) .
\end{aligned}
$$

Using the continuous embedding of $H^{\mu_{1}}(S)$ into $C^{1}(S)$ for $\mu_{1}>3 / 2$ and of $H^{\mu_{2}}(S)$ into $C(S)$ for $\mu_{2}>1 / 2$ and the inverse estimate $\|q\|_{H^{\mu}} \leq C\left(1+N^{2}\right)^{\mu / 2}\|q\|_{L^{2}}$ for $q \in T_{N}$, we conclude

$$
\frac{1}{N} \sum_{k=0}^{N-1}\left|\left(P_{N} g\right)(\hat{x})_{k}\right|^{2} \leq C(\mu)\left(1+\frac{\left(1+N^{2}\right)^{\mu}}{N}\right)\left\|P_{N} g\right\|_{L^{2}}^{2}
$$

Copyright $@$ by SIAM. Unauthorized reproduction of this article is prohibited. 
for any $\mu>1$. Observing $\left\|P_{N}\right\|=1$, choosing $s$ and $\mu$ appropriately, and combining (A.1) and (A.2) yields $\left\|\left(I-L_{N}\right) F_{\text {aux }}\right\| \rightarrow 0$ as $N \rightarrow \infty$. Note that we can achieve any order of convergence by choosing $s$ large enough.

It remains to prove $F\left(I-P_{N}\right) \rightarrow 0$, which we do by bounding $F q$ for $q \in T_{N}^{\perp}$, the orthogonal complement of $T_{N}$. We represent $q$ and $F q$ by their Fourier series, respectively $q=\sum_{|k| \geq N / 2} \alpha_{k} t_{k}$ and $F q=\sum_{l \in \mathbb{Z}} \gamma_{l} t_{l}$. Then, by partial integration,

$$
\begin{aligned}
\gamma_{l} & =\frac{1}{2 \pi} \int_{S} F q(\hat{x}) \overline{t_{l}(\hat{x})} d s(\hat{x})=\frac{1}{2 \pi} \int_{S} \int_{S} u^{\infty}(\hat{x}, \hat{y}) q(\hat{y}) \overline{t_{l}(\hat{x})} d s(\hat{y}) d s(\hat{x}) \\
& =\frac{1}{2 \pi} \sum_{|k| \geq N / 2} \alpha_{k} \int_{S} \int_{S} u^{\infty}(\hat{x}, \hat{y}) t_{k}(\hat{y}) \overline{t_{l}(\hat{x})} d s(\hat{y}) d s(\hat{x}) \\
& =\frac{-i}{2 \pi} \sum_{|k| \geq N / 2} \frac{\alpha_{k}}{k} \int_{S} \int_{S} \frac{\partial u^{\infty}(\hat{x}, \hat{y})}{\partial \hat{y}^{\perp}} t_{k}(\hat{y}) \overline{t_{l}(\hat{x})} d s(\hat{y}) d s(\hat{x}) .
\end{aligned}
$$

Here the symbol $\partial / \partial \hat{y}^{\perp}$ denotes a derivative in the angular direction with respect to $\hat{y}$. Consequently,

$$
\begin{aligned}
\left|\gamma_{l}\right|^{2} & \leq \frac{1}{(2 \pi)^{2}}\left(\sum_{|k| \geq N / 2}\left|\frac{\alpha_{k}}{k}\right|^{2}\right)\left(\sum_{|k| \geq N / 2}\left|\int_{S} \int_{S} \frac{\partial u^{\infty}(\hat{x}, \hat{y})}{\partial \hat{y}^{\perp}} t_{k}(\hat{y}) \overline{t_{l}(\hat{x})} d s(\hat{y}) d s(\hat{x})\right|^{2}\right) \\
& \leq \frac{1}{(\pi N)^{2}}\|q\|_{L^{2}}^{2}\left(\sum_{|k| \geq N / 2}\left|\int_{S} \int_{S} \frac{\partial u^{\infty}(\hat{x}, \hat{y})}{\partial \hat{y}^{\perp}} t_{k}(\hat{y}) \overline{t_{l}(\hat{x})} d s(\hat{y}) d s(\hat{x})\right|^{2}\right) .
\end{aligned}
$$

We now have the estimate

$$
\|F q\|_{L^{2}}^{2}=\sum_{l \in \mathbb{Z}}\left|\gamma_{l}\right|^{2} \leq \frac{1}{(\pi N)^{2}}\left\|\frac{\partial u^{\infty}(\hat{x}, \hat{y})}{\partial \hat{y}^{\perp}}\right\|_{L^{2}(S \times S)}^{2}\|q\|_{L^{2}}^{2}
$$

for all $q \in T_{N}^{\perp}$. As $\left\|I-P_{N}\right\|=1$, we have proved $F\left(I-P_{N}\right) \rightarrow 0$ as $N \rightarrow \infty$. Note again that we can, in fact, prove any order of convergence by carrying out further partial integrations with respect to $I-P_{N}$; i.e., convergence is superalgebraic.

\section{REFERENCES}

[1] C. B. Amar, N. Gmati, C. Hazard, and K. Ramdani, Numerical simulation of acoustic time reversal mirrors, SIAM J. Appl. Math., 67 (2007), pp. 777-791.

[2] H. Ammari, E. IAKovleva, And D. Lesselier, Two numerical methods for recovering small inclusions from the scattering amplitude at a fixed frequency, SIAM J. Sci. Comput., 27 (2005), pp. 130-158.

[3] L. Borcea, G. Papanicolaou, and F. G. Vasquez, Edge illumination and imaging of extended reflectors, SIAM J. Imaging Sci., 1 (2008), pp. 75-114.

[4] F. Cakoni and D. Colton, The linear sampling method for cracks, Inverse Problems, 19 (2003), pp. 279-295.

[5] M. Cheney, The linear sampling method and the MUSIC algorithm, Inverse Problems, 17 (2001), pp. 591-596.

[6] D. Colton And A. Kirsch, A simple method for solving inverse scattering problems in the resonance region, Inverse Problems, 12 (1996), pp. 383-393.

[7] D. L. Colton and R. Kress, Inverse Acoustic and Electromagnetic Scattering Theory, 2nd ed., Springer, New York, 1998.

[8] A. Devaney and E. Marengo, Nonradiating sources with connections to the adjoint problem, Phys. Rev. E, 70 (2004), paper 037601.

Copyright $@$ by SIAM. Unauthorized reproduction of this article is prohibited. 
[9] A. Devaney, E. Marengo, And F. Gruber, Time-reversal-based imaging and inverse scattering of multiply scattering point targets, J. Acoust. Soc. Amer., 118 (2005), pp. 3129-3138.

[10] P. Hartman And C. Wilcox, On solutions of the Helmholtz equation in exterior domains, Math Zeitschr., 75 (1961), pp. 228-255.

[11] C. HazARD AND K. RAMDAnI, Selective acoustic focusing using time-harmonic reversal mirrors, SIAM J. Appl. Math., 64 (2004), pp. 1057-1076.

[12] S. Hou, K. Solna, And H. ZhaO, A direct imaging algorithm for extended targets, Inverse Problems, 22 (2006), pp. 1151-1178.

[13] T. Kato, Perturbation Theory for Linear Operators, 2nd ed., Springer, New York, 1995.

[14] A. KIRSCH, Characterization of the shape of a scattering obstacle using the spectral data of the far field operator, Inverse Problems, 14 (1998), pp. 1489-1512.

[15] A. KIRSCH, Factorization of the far field operator for the inhomogeneous medium case and an application in inverse scattering theory, Inverse Problems, 15 (1999), pp. 413-429.

[16] A. KIRSCH, The MUSIC-algorithm and the factorization method in inverse scattering theory for inhomogeneous media, Inverse Problems, 18 (2002), pp. 1025-1040.

[17] A. Kirsch and N. Grinberg, The Factorization Method for Inverse Problems, Oxford Lect. Ser. Math. Appl. 36, Oxford University Press, London, 2008.

[18] A. KIRSCh AND S. RitTer, A linear sampling method for inverse scattering from an open arc, Inverse Problems, 16 (2000), pp. 89-105.

[19] R. KRess, Inverse scattering from an open arc, Math. Methods Appl. Sci., 18 (1995), pp. 183-219.

[20] R. Kress, Linear Integral Equations, 2nd ed., Springer, New York, 1999.

[21] A. Lechleiter, A regularization technique for the factorization method, Inverse Problems, 22 (2006), pp. 1605-1625.

[22] D. R. LukE, Multifrequency inverse obstacle scattering: The point source method and generalized filtered backprojection, Math. Comput. Simulation, 66 (2004), pp. 297-314.

[23] D. R. Luke And A. J. Devaney, Identifying scattering obstacles by the construction of nonscattering waves, SIAM J. Appl. Math., 68 (2007), pp. 271-291.

[24] D. R. Luke And R. Potthast, The point source method for inverse scattering in the time domain, Math. Methods Appl. Sci., 29 (2006), pp. 1501-1521.

[25] W. McLean, Strongly Elliptic Systems and Boundary Integral Operators, Cambridge University Press, Cambridge, UK, 2000.

[26] R. Potthast, A fast new method to solve inverse scattering problems, Inverse Problems, 12 (1996), pp. 731-742.

[27] R. SCHMIDT, Multiple emitter location and signal parameter estimation, IEEE Trans. Antennas and Propagation, 34 (1986), pp. 276-280.

Copyright (C) by SIAM. Unauthorized reproduction of this article is prohibited. 